\title{
Qualidade do sono em pacientes com apneia obstrutiva após cinco anos de uso de CPAP
}

\author{
Quality of sleep in patients with obstructive apnea after five years of CPAP use
}

\author{
Fabiana Lindemann Colvar Silveiraa, Gustavo Dias Ferreira ${ }^{b}$, Rafael Bueno Orcy ${ }^{b}$ \\ a Fisioterapeuta. Pós-graduada em Fisioterapia Cardiorrespiratória, Universidade Católica de Pelotas. \\ ${ }^{b}$ Fisioterapeuta. Doutor em Fisiologia. Professor da Universidade Federal de Pelotas.
}

RESUMO

Introdução: A Síndrome da Apneia/Hipopneia Obstrutiva do Sono (SAHOS) é caracterizada pela obstrução recorrente das vias aéreas superiores durante o sono. O tratamento de escolha é a aplicação de pressão positiva continua nas vias aéreas (CPAP) por máscara nasal. Apesar de extremamente efetivo, a adesão ao CPAP é variável, pois o fluxo contínuo de ar nas vias aéreas pode ocasionar desconforto, e muitas vezes resultar na interrupção do tratamento.

Objetivos: Comparar se há melhora do estado de sonolência diurna, da qualidade do sono, da composição corporal e do risco para apneia após cinco anos da utilização de CPAP.

Materiais e Métodos: Estudo longitudinal, com acompanhamento de 11 pacientes (9 homens, idade média de $63,73 \pm 9,1)$, com observações prévias ao uso do CPAP e após utilizarem durante cinco anos. Foram avaliadas a sonolência diurna (escala de Epworth), a qualidade do sono (escala visual), além de variáveis antopométricas, e da escala de Berlin (verificar maior risco de apneia). Dados foram analisados pelos testes t pareado e Wilcoxon.

Resultados: A análise da escala de Epworth mostrou uma diminuição do nível de sonolência diurna, assim como houve melhora no relato da qualidade do sono, no entanto variáveis antropométricas e o risco de apneia foram semelhantes após cinco anos de tratamento.

Conclusões: O uso do CPAP por um período contínuo é uma intervenção que melhora a qualidade de sono e níveis de sonolência diurna e, apesar de não interferir na massa corporal, pode ser uma estratégia importante na vida de pacientes com apneia do sono.

Palavras-chaves: ventilação não invasiva - cpap; síndromes da apneia do sono; fisioterapia.

\section{ABSTRACT}

Introduction: Obstructive Sleep Apnea/Hypopnea Syndrome is characterized by recurrent obstruction of the upper airways during sleep. The treatment is the application of continuous positive airway pressure (CPAP) through a nasal mask. Although extremely effective, adherence to CPAP is variable, as the continuous flow of air in the airways can cause discomfort, and often result in discontinuation of treatment.

Objective: To compare if there is improvement in the state of daytime sleepiness, sleep quality, body composition and risk for apnea after five years of CPAP use.

Materials and Methods: This was a longitudinal, descriptive, prospective study with follow-up of 11 patients (9 male, mean age of $63.73 \pm 9.1$ ), with observations previous to CPAP use and after using it for five years. Daytime sleepiness (Epworth scale), sleep quality (visual scale), in addition to antopometric variables, and the Berlin scale (to verify risk of apnea) were evaluated. Data were analyzed by paired t test and the Wilcoxon test.

Results: The analysis of the Epworth scale showed a decrease in the level of daytime sleepiness, as well as an improvement in the sleep quality, although anthropometric variables and the risk of apnea were similar after five years of CPAP use.

Conclusions: The use of CPAP for a continuous period is an intervention that improves sleep quality and diurnal sleepiness levels and, although it does not interfere with body mass, may be an important strategy in the life of patients with sleep apnea syndrome.

Keywords: nonivasive ventilation - cpap; sleep apnea syndromes; physiotherapy.

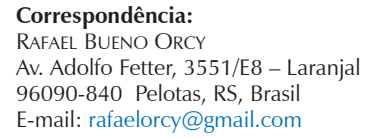




\section{INTRODUÇÃO}

O ronco, queixa de $40 \%$ a $60 \%$ dos adultos, é o ruído resultante da vibração da cavidade oral em decorrência da obstrução parcial das vias aéreas superiores, e está presente em 90\% a 95\% dos casos de Síndrome da Apneia/Hipopneia Obstrutiva do Sono (SAHOS) ${ }^{1}$. A prevalência da SAHOS é de 9\% da população masculina de meia idade (30 a 60 anos) e de $4 \%$ da população feminina após a menopausa, sendo ainda maior em faixas etárias avançadas e em indivíduos obesos $^{2}$.

A SAHOS é caracterizada pela obstrução completa ou parcial recorrente nas vias aéreas durante o sono, que causa períodos de apneia, dessaturação de hemoglobina e despertares frequentes, consequentemente sonolência diurna. Os episódios de apneia presentes nos indivíduos com SAHOS são decorrentes do relaxamento fisiológico da musculatura faríngea durante o sono e isso estreita e/ ou obstrui de forma completa as vias aéreas superiores ${ }^{3,4,5}$. A SAHOS está associada à morbidade e mortalidade cardiovascular, isto é, hipertensão arterial, angina, doenças isquêmicas e acidente vascular cerebral, sendo o infarto agudo do miocárdio a causa mais frequente de morte na SAHOS $^{3}$.

O tratamento de escolha para SAHOS moderada e grave é a aplicação noturna de pressão positiva continua nas vias aéreas (CPAP) através do uso de máscara nasal. O CPAP nasal virtualmente elimina os eventos obstrutivos respiratórios assim que uma pressão adequada é estabelecida, melhorando qualidade do sono. Apesar de extremamente efetivo, a adesão ao CPAP é variável, sendo a intolerância à mascara um problema clínico comum ${ }^{6,7}$. Dados indicam que pacientes com menores dimensões cefalométricas e com fraqueza de tecidos moles orofagíngeo tendem a ter uma menor adesão ao tratamento, e estes dois fatores estão fortemente associados com o sobrepeso e a obesidade, característica altamente prevalente na população com $\mathrm{SAHOS}^{6,7}$. Nestes casos, é essencial o trabalho do profissional fisioterapeuta para treinamento, adaptação e aumentar a persistência do pacientes, para que o tratamento seja continuado e obtenha sucesso ${ }^{7,8}$.

Já é claro na literatura que a SAHOS é uma condição altamente prevalente que afeta pessoas de todas as idades, levando a sérios problemas de saúde pública. Como altera qualidade de sono e influencia no bem estar do paciente, já está associada com doenças cardiovasculares, síndrome metabólica, comprometimento neurocognitivo e aumento dos acidentes automobilísticos ${ }^{8}$. Sabendo que o CPAP é o tratamento de escolha, justifica-se este estudo para verificar padrões de sonolência diária, qualidade do sono, riscos e padrões antropométricos em pacientes tratados e acompanhados por 5 anos.

O objetivo deste trabalho é comparar se há melhora do estado de sonolência diurna, da qualidade do sono, da composição corporal e do risco para apneia após cinco anos da utilização de CPAP em pacientes com Síndrome de Apneia/Hipopneia Obstrutiva do Sono.

\section{MATERIAIS E MÉTODOS}

Foi realizado um estudo prospectivo, longitudinal por cinco anos, em pacientes que foram diagnosticados com SAHOS por polissonografia em uma clínica especializada em Distúrbios do Sono na cidade de Pelotas-RS. Sendo uma primeira avaliação em 2010, previamente a utilização de CPAP, e outra em 2015, após cinco anos de tratamento noturno com CPAP domiciliar.

Este trabalho foi aprovado pelo Comitê de Ética em Pesquisa da Universidade Católica de Pelotas com o número de protocolo 2009/72, e os pacientes assinaram o Termo de Consentimento Livre e Esclarecido, concordando com a participação no estudo.

O cálculo do tamanho da amostra foi realizado considerando um desvio padrão de 5,8 na escala de Epworth ${ }^{6}$, utilizando-se uma diferença entre as médias de 5 pontos na escala, com poder estatístico de $80 \%$ e nível de significância de 0,05 . Foi utilizado o programa estatístico PEPI 404X e o número do tamanho da amostra foi calculado em 10 pacientes.

A amostra foi selecionada por conveniência e incluiu 11 pacientes (valor calculado $+10 \%$ ). Os critérios para inclusão foram pacientes da clínica que estavam iniciando o tratamento para SAHOS com CPAP domiciliar. Como exclusão, somente pacientes que não tinham indicação clara para uso do CPAP ou que apresentassem alguma lesão ortopédica de face. A idade destes pacientes variou entre 53 e 81 anos (com média de aproximadamente 64 anos), 9 eram do sexo masculino, e, corroborando com a maior prevalência nesta condição, todos estavam acima do peso recomendado (4 pacientes com sobrepeso e 7 com obesidade na primeira avaliação). Foram realizadas coletas e avaliações prévias ao uso do CPAP no ano de 2010, e reavaliações com os mesmos instrumentos e realizadas pela mesma pesquisadora (fisioterapeuta proprietária da clínica) em 2015. É importante mencionar, que apesar de todos os pacientes terem utilizado a interface nasal, a indicação do tratamento do CPAP foi clínica e independente do estudo, portanto, o tipo de CPAP e as pressões utilizadas não foram controladas, e foram prescritas de forma individualizada, de acordo com a necessidade 
do paciente. A recomendação para o tratamento era que o uso fosse em todas as noites e teve uma pressão que variou entre 10 e $12 \mathrm{cmH}_{2} \mathrm{O}$. Para o estudo, era importante que o paciente realizasse seu tratamento pelo período de 5 anos, e assim, pudéssemos avaliar as variáveis que foram propostas. Como a utilização do instrumento era parte do tratamento, os pacientes tinham consultas regulares na clínica para ajustes de parâmetros e acompanhamento clínico.

Para pesquisa, foram aplicadas as escalas de Epworth ${ }^{6}$, que avalia o grau de sonolência excessiva diurna (escala varia de 0 a 24 pontos, sendo que pontuação maior que 9 é indicativo de sonolência excessiva diurna), e escala de Berlin, com avaliação em 3 diferentes categorias, na qual os indivíduos que se enquadram em pelo menos duas categorias são considerados de alto risco para SAHOS 9 . Estes questionários são curtos e com perguntas simples, a maioria de múltipla escolha, e já está bem descrito na literatura como instrumento para avaliação de chance de cochilar durante o dia (sonolência diurna) e risco para apneia do sono, respectivamente ${ }^{6,9}$. Também foram coletados dados de qualidade do sono por escala visual simples (sendo 0 muito ruim e 10 ótimo), variáveis antropométricas, como peso e altura, e dados secundários de hábitos de estilo de vida (se praticava atividade física - "Você realiza atividade física pelo menos 150 minutos por semana?" e tabagismo - "Atualmente, você fuma?"), além da satisfação com uso do CPAP (sendo 0 para insatisfeito e 10 muito satisfeito).

Após realização do teste de Shapiro-Wilk, os dados foram apresentados de forma descritiva, utilizando-se média (desvio padrão) para variáveis paramétricas e mediana (intervalo interquartil 25\%-75\%) para variável não paramétricas. Foram utilizados os testes estatísticos t pareado (variáveis paramétricas) e Wilcoxon (variáveis não-paramétricas). Os dados foram tabelados no Excel e analisados utilizando o pacote estatístico do SPSS 20.0.

\section{RESULTADOS}

A amostra foi composta por 11 indivíduos (9 do sexo masculino), que em 2010 tinham idade média de 63,7 799,1 anos. Em relação as medidas antropométricas, o índice de massa corporal (IMC) médio foi de $31,7 \pm 3,8 \mathrm{~kg} / \mathrm{m}^{2}$ na primeira coleta (2010) e de $32,9 \pm 3,8 \mathrm{~kg} / \mathrm{m}^{2}$ na segunda coleta (2015), mantendo as características de composição corporal dos pacientes no intervalo de cinco anos $(p=0,242)$. Destes, 4 pacientes apresentam sobrepeso, 4 obesidade grau I e 3 obesidade grau II, não alterando a classe com o passar do tempo. Não houve perdas, apenas 1 paciente interrompeu o uso de CPAP por um mês contínuo durante os 5 anos, porém ele não foi excluído do estudo por novamente ter aderido ao tratamento no período seguinte. Assim, acreditamos que para uma intervenção de cinco anos a referida pausa não tenha interferido nos resultados.

Sobre a qualidade de sono, a mediana (intervalo interquartil) de pontuação na escala visual em 2010 foi de 8 (8-10) e depois de cinco anos, em 2015, foi de 10 (8-10). Pelo teste de Wilcoxon pareado (signed-ranks test), o valor do teste estatístico (10) foi igual ao valor crítico (10), rejeitando hipótese de igualdade, mostrando diferença estatística $(p=0,026)$, indicando melhora nesta variável.

Em relação a sonolência diurna, a mediana da escala de Epworth antes (2010) foi de 9 (3-10), sendo que 3 pacientes pontuaram até 12 (o que indica que, além do risco, necessitam procurar ajuda do especialista do sono) e 4 pacientes fizeram 3 pontos, estando dentro da normalidade. Após tratamento com CPAP por 5 anos (2015), a mediana baixou para 4 (3-10). Novamente utilizando o signed-ranks test, o valor do teste estatístico (5) foi menor que o valor crítico do teste (10), rejeitando hipótese de igualdade, mostrando diferença estatística $(p=0,007)$, e confirmando melhora nessa variável. Estes resultados indicam melhora tanto da qualidade de sono noturno, como da menor sonolência durante o dia.

Comparando a escala de Berlim, antes e depois, obteve-se mediana 1 em ambos os tempos (normalmente relacionado com a pontuação na categoria 3: presença de pressão alta ou IMC acima de $30 \mathrm{~kg} / \mathrm{m}^{2}$ ), apresentando risco pequeno de obstrução completa durante o sono, que permaneceu com uso do CPAP $(p=0,589)$.

Dados complementares mostram que dos 11 pacientes da amostra, 9 possuíam algum tipo de doença crônica que necessitasse tratamento com medicação, em geral hipertensão e/ou diabetes tipo II, provavelmente relacionados com o alto IMC, o que pode agravar o risco de apneia (indicado pelo questionário de Berlin). Por outro lado, nenhum era tabagista e 5 mencionaram que realizam atividade física de forma regular e em intensidade moderada, fatores protetores que auxiliam o tratamento.

Em relação ao acompanhamento, o tempo de uso da ventilação mecânica não invasiva é de apenas 5 anos (início em 2010) e indicado para uso domiciliar para tratamento clínico individualizado. Sendo que 4 pacientes utilizam CPAP S8, 4 utilizam CPAP S7 e 3 utilizam CPAP Tango, ainda, três utilizaram umidificador. Após 5 anos de utilização, 2 pacientes disseram estar satisfeito com o uso do CPAP (pontuaram 8 e 9 na escala de qualidade de sono) e 9 pacientes relataram sentir-se muito satisfeito (pontuando 10).

Estes dados estão apresentados na Tabela $\mathbf{1}$. 
Tabela 1. Características da amostra em um intervalo de 5 anos.

\begin{tabular}{|c|c|c|c|}
\hline & 2010 & 2015 & Valor de $p$ \\
\hline Idade $(\operatorname{anos})^{a}$ & $63,73 \pm 9,1$ & $69,01 \pm 9,0$ & 0,212 \\
\hline Peso $(k g)^{a}$ & $89,05 \pm 11,27$ & $92,45 \pm 12,64$ & 0,209 \\
\hline Altura (metros) ${ }^{\mathrm{a}}$ & $1,67 \pm 0,07$ & $1,67 \pm 0,07$ & 1,000 \\
\hline $\mathrm{IMC}\left(\mathrm{kg} / \mathrm{m}^{2}\right)^{\mathrm{a}}$ & $31,77 \pm 3,83$ & $32,95 \pm 3,86$ & 0,242 \\
\hline Grau de satisfação (escala 0-10) ${ }^{b}$ & - & $10(10-10)$ & - \\
\hline Qualidade de sono (escala 0-10) ${ }^{\mathrm{b}}$ & $8(8-10)$ & $10(8-10)$ & $0,026^{*}$ \\
\hline Escala de Epworth (escala 0-24) ${ }^{b}$ & $9(3-10)$ & $4(3-10)$ & $0,007^{*}$ \\
\hline Escala de Berlin (0-3 categorias +$)^{b}$ & $1(1-1)$ & $1(0-1)$ & 0,589 \\
\hline
\end{tabular}

amédia \pm desvio padrão; ${ }^{b}$ mediana (IQ 25-75\%).

*Diferença significativa pelo teste de Wilcoxon.

\section{DISCUSSÃO}

Este estudo mostra que a utilização contínua de CPAP melhora estatisticamente a qualidade do sono e a sonolência diurna, apesar de não alterar riscos para apneia e composição corporal. A amostra foi predominantemente masculina, sendo o sexo com maior fator de risco para SAHOS. A SAHOS tem se configurado como um problema de saúde para grande parte da população adulta. Estima-se que cerca de $2 \%$ da população feminina e $9 \%$ da população masculina, entre 40 e 60 anos, apresentam essa síndrome ${ }^{10,11}$.

A apneia é definida como uma cessação completa de fluxo oronasal durante 10 segundos ou mais. A hipopneia se caracteriza por uma redução de no mínimo 50\% no fluxo oronasal durando 10 segundos ou mais. As apneias podem ser classificadas como obstrutivas, mistas ou centrais. Nas apneias obstrutivas, o fluxo aéreo é impedido pelo colapso das vias aéreas superiores, apesar dos esforços repetidos para restabelecer a respiração. Durante as apneias centrais, a ventilação cessa porque o sistema nervoso central é incapaz de ativar o diafragma e ou músculos respiratórios. As apneias mistas começam com uma pausa do centro respiratório, seguido por aumento sucessivo do esforço respiratório contra uma via aérea obstruída ${ }^{4,5}$. Neste estudo, os pacientes eram portadores de apneias obstrutivas, as quais respondem melhor a intervenção de pressão positiva na via aérea.

Um estudo recente realizado por pesquisadores no Reino Unido avaliou o custo-benefício do CPAP em pacientes com SAHOS por 14 anos na perspectiva do Serviço Nacional de Saúde (NHS). Os resultados demonstraram que após 2 anos de tratamento houve um aumento da probabilidade de sobrevivência em $25 \%$, diminuição do risco relativo de evento cardiovascular em $46 \%$, diminuição do risco relativo de acidente vascular cerebral em $49 \%$, aumento da probabilidade de sobrevivência sem eventos em 92\% ${ }^{11}$. Sabendo destes benefícios, o presente estudo fez um acompanhamento de uso da ventilação mecânica não-invasiva por 5 anos, para avaliar fatores relacionados diretamente com as principais queixas deste tipo de paciente: a qualidade do sono e a sonolência diária, além de fatores de risco importantes para pior prognóstico: massa corporal e risco de eventos de apneia. Os resultados foram encorajadores, melhorando qualidade de sono e diminuindo sonolência diária medidos por instrumentos validados, assim, consequentemente, pode-se extrapolar para uma melhora na qualidade de vida.

A obesidade é reconhecida como o principal fator de risco para SAHOS, assumindo esse papel especial por estar presente em aproximadamente $70 \%$ dos pacientes apneicos ${ }^{12,13}$. Conforme o perfil obeso desta população, pode-se sugerir que o grau de apneia é pior em pacientes com obesidade mórbida, associando de forma direta e crescente a síndrome de obesidade com a hipoventilação. O baixo volume respiratório é ainda mais evidente no período noturno, condicionando a diminuição da concentração de oxigênio no sangue arterial, com alterações da estrutura e fases do sono e da sonolência diurna ${ }^{14}$. Um recente estudo indicou que o é necessário utilizar técnicas de pressão positiva em pacientes obesos, para melhor recrutamento pulmonar e manter os alvéolos abertos ${ }^{13,15}$. Esta é uma característica muito comum dos pacientes com SAHOS, e neste acompanhamento, apesar de relato da melhora no sono, os pacientes permaneceram com sobrepeso/obesidade durante os 5 anos.

A escala de sonolência excessiva foi idealizada com base em observações à natureza e a ocorrência da sonolência diurna. Trata-se de um questionário autoaplicável que avalia a probabilidade de adormecer em oito situações envolvendo atividades diárias. O escore varia de 0 a 24 , sendo que o escore acima de 9 sugere o diagnóstico da 
sonolência excessiva diurna ${ }^{16}$. Em um recente ensaio clínico randomizado, de acompanhamento de 12 meses, pacientes que utilizaram CPAP tiveram menor pontuação na avaliação de sonolência diária, avaliada pela escala de Epworth, além de terem menores gastos com sistema de saúde neste período, mostrando o ótimo custo-benefício do tratamento ${ }^{17}$. Nesta avaliação, o escore da escala de Epworth diminuiu nas medidas antes de depois, onde no ano de 2010, a mediana de pontos dos pacientes estava no ponto de corte de 9, baixando para 4 após a intervenção de cinco anos com CPAP. Clinicamente, isto indica uma importante diminuição da sonolência excessiva diurna, e pode estar associado ao aumento da qualidade do sono noturno, mencionada pelos pacientes no questionário de escala visual, o qual aumentou da mediana 8 para 10 no decorrer dos cinco anos, além do excelente grau de satisfação dos pacientes com o tratamento, indicando a melhora da SAHOS.

O Questionário de Berlim é um dos mais reconhecidos instrumentos de rastreio utilizado para detectar alto ou baixo índice de SAHOS. Este questionário inclui 10 itens, organizados em 3 categorias referentes à roncopatia e apneias presenciadas (5 itens), sonolência diurna (4 itens) e hipertensão arterial/obesidade (1 item). Informação sobre o sexo, idade, altura, peso, circunferência do pescoço e raça é também solicitada. A determinação do alto ou baixo risco para gravidade da SAHOS é baseada nas respostas em cada categoria de itens. Portanto, duas ou mais categorias positivas indicam alto risco para SAHOS ${ }^{18}$. Os valores da escala de Berlim permaneceram baixo após cinco anos do uso do CPAP, na maioria dos casos sendo positivo em apenas 1 categoria, em geral, a presença da obesidade e/ou hipertensão arterial.

Como limitação do estudo, não foi possível controlar os parâmetros do CPAP, visto que a prescrição foi clínica e individual, independente do estudo. Pelo mesmo motivo, este estudo longitudinal não contou com grupo controle e/ou utilização de placebo, pois todos os participantes foram incluídos na pesquisa por estarem em tratamento para SAHOS com CPAP domiciliar. Ainda, a utilização de questionários subjetivos, que apesar de serem validados na literatura, o paciente pode responder de forma positiva por não ser cegado ao tratamento. Para o cálculo do tamanho da amostra, utilizou-se valores relacionados apenas com a variável da escala de Epworth, assim, sugere-se um estudo de intervenção randomizado com maior número de participantes e grupo controle para fortalecer estes dados.

Com isso, pode-se concluir que apesar de o risco para apneia e a composição corporal não terem se modificado durante o acompanhamento (permanecendo o perfil de sobrepeso e obesidade nestes pacientes), neste estudo, os indicadores de sonolência diária e de qualidade de sono melhoraram após cinco anos de uso de CPAP, ficando evidente a satisfação dos pacientes SAHOS com o tratamento noturno com a ventilação não-invasiva.

\section{REFERÊNCIAS}

1. Balbani APS, Formigoni GGS. Ronco e síndrome da apnéia obstrutiva do sono. Rev Ass Med Bras. 1999;45(3):273-8. https:// doi.org/10.1590/S0104-42301999000300013

2. Palombini LO. Critérios diagnósticos e tratamento dos distúrbios respiratórios do sono: RERA. J. Bras Pneumol. 2010;36(supl.2):S1-S61. https://doi.org/10.1590/S1806-37132010001400002

3. Palombini LO. Fisiopatologia dos distúrbios respiratórios do sono. J Bras Pneumol. 2010;36(supl. 2):S1-S61. https://doi.org/10.1590/ S1806-37132010001400002

4. Sleep-related breathing disorders in adults: recommendations for syndrome definition and measurement techniques in clinical research. The Report of an American Academy of Sleep Medicine Task Force. Sleep. 1999;22(5):667-89. https://doi.org/10.1093/ sleep/22.5.667

5. Rühle KH, Schlenker E, Randerath W. Upper airway resistance syndrome. Respiration. 1997;64(Suppl. 1):29-34. https://doi. org/10.1159/000196733

6. Dempsey JA, Skatrud JB, Jacques AJ, Ewanowski SJ, Woodson BT, Hanson PR, Goodman B. Anatomic determinants of sleep-disordered breathing across the spectrum of clinical and nonclinical male subjects. Chest. 2002;122(3):840-51. 7. Friedman M, Hamilton C, Samuelson CG, Lundgren ME, Pott T. Diagnostic value of the Friedman Tongue position and Mallampati classification for obstructive sleep apnea: a meta-analysis. Otolaryngol Head Neck Surg. 2013;148(4):540-7. https://doi.org/10.1177/0194599812473413

8. Cao MT, Sternbach JM, Guilleminault C. Continuous positive airway pressure therapy in obstructive sleep apnea: benefits and alternatives. Expert Rev Respir Med. 2017;11(4):259-72. https:// doi.org/10.1080/17476348.2017.1305893

9. Remmers JE, deGroot WJ, Sauerland EK, Anch AM. Pathogenesis of upper airway occlusion during sleep. J Appl Physiol Respir Environ Exerc Physiol. 1978;44(6):931-8.

10. Almeida MAO, Teixeira AOB, Vieira LS, Quintão CCA. Tratamento da síndrome da apnéia e hipopnéia obstrutiva do sono com aparelhos intrabucais. Rev Bras Otorrinolaringol. 2006;72(5)200-6. https://doi.org/10.1590/S0034-72992006000500018

11. uest JF, Helter MT, Morga A, Stradling JR. Cost-effectiveness of using continuous positive airway pressure in the treatment of severe obstructive sleep apnoea/hypopnoea syndrome in the UK. Thorax. 2008;63(10):860-5. https://doi.org/10.1136/thx.2007.086454

12. Mancini MC, Aloe F, Tavares S. Apnéia do sono em obesos. Arq Bras Endocrinol Metab. 2000;44(1):81-90. https://doi.org/10.1590/ S0004-27302000000100013

13. Zhang C, Pirrone M, Imber DA, Ackman JB, Kacmarek RM, Berra L. Optimization of Mechanical Ventilation in a 31-Year-Old Morbidly Obese Man With Refractory Hypoxemia. A A Case Rep. 2017;8(1):7-10. https://doi.org/10.1213/XAA.0000000000000408 
14. Carneiro G, Ribeiro Filho FF, Togeiro SM, Tufik S, Zanella MT. Interações entre síndrome da apnéia do sono e resistência à insulina. Arq Bras Endocrinol Metab. 2007;7(51):1035-40. https:// doi.org/10.1590/S0004-27302007000700003

15. Pirrone M, Fisher D, Chipman D, Imber DA, Mietto C, Berra L. Recruitment Maneuvers and Positive End-Expiratory Pressure Titration in Morbidly Obese ICU Patients. Crit Care Med. 2016;44(2):300-7. https://doi.org/10.1097/CCM.0000000000001387

16. Bertolazi AN, Fagondes SC, Hoff LS, Pedro VD, Menna Barreto SS, Johns MW. Validação da escala de sonolência de Epworth em português para uso no Brasil. J Bras Pneumol. 2009;9(45):878.
17. McMillan A, Bratton DJ, Faria R, Laskawiec-Szkonter M, Griffin S, Davies RJ, Nunn AJ, Stradling JR, Riha RL, Morrell MJ; PREDICT Investigators. Continuous positive airway pressure in older people with obstructive sleep apnoea syndrome (PREDICT): a 12-month, multicentre, randomised trial. Lancet Respir Med. 2014;2(10):804-12. https://doi.org/10.1016/S2213-2600(14) 70172-9

18. Netzer NC, Stoohs RA, Netzer CM, Clark K, Strohl KP. Using the Berlin Questionnaire to identify patients at risk for the sleep apnea syndrome. Ann Intern Med. 1999;131(7):485-91. https://doi. org/10.7326/0003-4819-131-7-199910050-00002 